\title{
Approximation of classes of periodic functions of several variables with given majorant of mixed moduli of continuity
}

\author{
Fedunyk-Yaremchuk O.V. ${ }^{凶}$, Hembars'ka S.B.
}

\begin{abstract}
In this paper, we continue the study of approximation characteristics of the classes $B_{p, \theta}^{\Omega}$ of periodic functions of several variables whose majorant of the mixed moduli of continuity contains both exponential and logarithmic multipliers. We obtain the exact-order estimates of the orthoprojective widths of the classes $B_{p, \theta}^{\Omega}$ in the space $L_{q}, 1 \leq p<q<\infty$, and also establish the exact-order estimates of approximation for these classes of functions in the space $L_{q}$ by using linear operators satisfying certain conditions.

Key words and phrases: orthoprojective width, mixed modulus of continuity, linear operator, Vallée-Poussin kernel, Fejér kernel.
\end{abstract}

\footnotetext{
Lesya Ukrainka Volyn National University, 13 Voli avenue, 43025, Lutsk, Ukraine

$\checkmark$ Corresponding author

E-mail: fedunyk.○.v@gmail.com (Fedunyk-Yaremchuk O.V.), gembarskaya 72@gmail.com (Hembars'ka S.B.)
}

\section{Introduction}

Let $\mathbb{R}^{d}, d \geq 1$, denotes the $d$-dimensional space with elements $x=\left(x_{1}, \ldots, x_{d}\right)$. In what follows $(x, y)=x_{1} y_{1}+\ldots+x_{d} y_{d}$ stands for scalar product. Let $L_{p}\left(\pi_{d}\right), 1 \leq p<\infty$, be the space of functions $f(x)=f\left(x_{1}, \ldots, x_{d}\right)$, which are $2 \pi$-periodic in each variable and summable in degree $p$ on the cube $\pi_{d}=\prod_{j=1}^{d}[0 ; 2 \pi]$, endowed with the norm

$$
\|f\|_{p}:=\|f\|_{L_{p}\left(\pi_{d}\right)}=\left((2 \pi)^{-d} \int_{\pi_{d}}|f(x)|^{p} d x\right)^{1 / p} .
$$

Let $L_{\infty}\left(\pi_{d}\right)$ be the space of essentially bounded functions $f(x)=f\left(x_{1}, \ldots, x_{d}\right)$, which are $2 \pi$-periodic in each variable, with the norm

$$
\|f\|_{\infty}:=\|f\|_{L_{\infty}\left(\pi_{d}\right)}=\underset{x \in \pi_{d}}{\operatorname{ess} \sup }|f(x)| .
$$

Further, we assume that for functions $f \in L_{p}\left(\pi_{d}\right)$ the following additional condition holds

$$
\int_{0}^{2 \pi} f(x) d x_{j}=0, \quad j=\overline{1, d} .
$$

For $f \in L_{p}\left(\pi_{d}\right), 1 \leq p \leq \infty$, and $t=\left(t_{1}, \ldots, t_{d}\right), t_{j} \geq 0, j=\overline{1, d}$, we consider the mixed modulus of continuity of the order $l$

$$
\Omega_{l}(f, t)_{p}=\sup _{\left|h_{j}\right| \leq t_{j}, j=\overline{1, d}}\left\|\Delta_{h}^{l} f(\cdot)\right\|_{p},
$$

$\mathrm{y} \Delta \mathrm{K} 517.51$

2020 Mathematics Subject Classification: 42B99. 
where $l \in \mathbb{N}, \Delta_{h}^{l} f(x)=\Delta_{h_{1}}^{l} \ldots \Delta_{h_{d}}^{l} f(x)=\Delta_{h_{d}}^{l}\left(\ldots\left(\Delta_{h_{1}}^{l} f(x)\right)\right)$ is a mixed difference of the order $l$ with a vector step $h=\left(h_{1}, \ldots, h_{d}\right)$, and the difference of the $l$ th order with a step $h_{j}$ in the variable $x_{j}$ is defined as follows

$$
\Delta_{h_{j}}^{l} f(x)=\sum_{n=0}^{l}(-1)^{l-n} C_{l}^{n} f\left(x_{1}, \ldots, x_{j-1}, x_{j}+n h_{j}, x_{j+1}, \ldots, x_{d}\right) .
$$

Let $\Omega(t)=\Omega\left(t_{1}, \ldots, t_{d}\right)$ be a given function of the type of a mixed modulus of continuity of the order $l$, which satisfies the following conditions:

1) $\Omega(t)>0$ if $t_{j}>0, j=\overline{1, d}$, and $\Omega(t)=0$ if $\prod_{j=1}^{d} t_{j}=0$;

2) $\Omega(t)$ is nondecreasing in each variable;

3) $\Omega\left(m_{1} t_{1}, \ldots, m_{d} t_{d}\right) \leq\left(\prod_{j=1}^{d} m_{j}\right)^{l} \Omega(t)$ for $m_{j} \in \mathbb{N}, j=\overline{1, d}$;

4) $\Omega(t)$ is continuous for $t_{j} \geq 0, j=\overline{1, d}$.

We assume that $\Omega(t)$ satisfies also the conditions $(S)$ and $\left(S_{l}\right)$, which are called the BariStechkin conditions [1]. This means the following.

A function of one variable $\varphi(\tau) \geq 0$ satisfies the condition $(S)$ if $\varphi(\tau) / \tau^{\alpha}$ almost increases for some $\alpha>0$, i.e. there exists a constant $C_{1}>0$ independent of $\tau_{1}$ and $\tau_{2}$ such that

$$
\frac{\varphi\left(\tau_{1}\right)}{\tau_{1}^{\alpha}} \leq C_{1} \frac{\varphi\left(\tau_{2}\right)}{\tau_{2}^{\alpha}}, \quad 0<\tau_{1} \leq \tau_{2} \leq 1
$$

A function $\varphi(\tau) \geq 0$ satisfies the condition $\left(S_{l}\right)$ if $\varphi(\tau) / \tau^{\gamma}$ almost decreases for some $0<\gamma<l$, i.e. there exists a constant $C_{2}>0$ independent of $\tau_{1}$ and $\tau_{2}$ such that

$$
\frac{\varphi\left(\tau_{1}\right)}{\tau_{1}^{\gamma}} \geq C_{2} \frac{\varphi\left(\tau_{2}\right)}{\tau_{2}^{\gamma}}, \quad 0<\tau_{1} \leq \tau_{2} \leq 1
$$

In the case of $d>1$ we say that $\Omega(t), t \in \mathbb{R}_{+}^{d}$, satisfies the conditions $(S)$ and $\left(S_{l}\right)$ if $\Omega(t)$ satisfies these conditions in each variable $t_{j}$ for fixed $t_{i}, i \neq j$.

Thus, let $1 \leq p \leq \infty, 1 \leq \theta \leq \infty$, and let $\Omega(t)$ be a given function of the type of a mixed modulus of continuity of the order $l$. Then the classes $B_{p, \theta}^{\Omega}$ are defined in the following way [22]:

$$
B_{p, \theta}^{\Omega}=\left\{f \in L_{p}\left(\pi_{d}\right):\|f\|_{B_{p, \theta}^{\Omega}} \leq 1\right\},
$$

where

$$
\|f\|_{B_{p, \theta}^{\Omega}}=\left\{\int_{\pi_{d}}\left(\frac{\Omega_{l}(f, t)_{p}}{\Omega(t)}\right)^{\theta} \prod_{j=1}^{d} \frac{d t_{j}}{t_{j}}\right\}^{1 / \theta}, \quad 1 \leq \theta<\infty, \quad\|f\|_{B,, \infty}^{\Omega}=\sup _{t>0} \frac{\Omega_{l}(f, t)_{p}}{\Omega(t)},
$$

(the expression $t>0$ for $t=\left(t_{1}, \ldots, t_{d}\right)$ is equivalent to $t_{j}>0, j=\overline{1, d}$ ).

We note that for $\theta=\infty$ the classes $B_{p, \theta}^{\Omega}$ coincide with the classes $H_{p}^{\Omega}$, which were considered by N.N. Pustovoitov in [14].

In the subsequent, it will be convenient to use the equivalent (to within absolute constants) definition of the classes $B_{p, \theta}^{\Omega}$. For this purpose, we need the corresponding notations. 
To every vector $s=\left(s_{1}, \ldots, s_{d}\right), s_{j} \in \mathbb{N}, j=\overline{1, d}$, we put in correspondence the set

$$
\rho(s)=\left\{k=\left(k_{1}, \ldots, k_{d}\right): 2^{s_{j}-1} \leq\left|k_{j}\right|<2^{s_{j}}, k_{j} \in \mathbb{Z}, j=\overline{1, d}\right\},
$$

and for $f \in L_{p}\left(\pi_{d}\right), 1<p<\infty$, we denote

$$
\delta_{s}(f):=\delta_{\mathcal{S}}(f, x)=\sum_{k \in \rho(s)} \widehat{f}(k) e^{i(k, x)},
$$

where

$$
\widehat{f}(k)=(2 \pi)^{-d} \int_{\pi_{d}} f(t) e^{-i(k, t)} d t
$$

are the Fourier coefficients of the function $f$.

Let $1<p<\infty, 1 \leq \theta \leq \infty$, and let $\Omega(t)$ be a given function of the type of a mixed modulus of continuity of the order $l$ that satisfies the conditions 1$)-4),(S)$ and $\left(S_{l}\right)$. Then, to within absolute constants, the classes $B_{p, \theta}^{\Omega}$ can be defined as follows [22]:

$$
B_{p, \theta}^{\Omega}=\left\{f \in L_{p}\left(\pi_{d}\right):\|f\|_{B_{p, \theta}^{\Omega}}=\left(\sum_{s} \Omega^{-\theta}\left(2^{-s}\right)\left\|\delta_{s}(f)\right\|_{p}^{\theta}\right)^{1 / \theta} \leq 1\right\} \quad \text { for } 1 \leq \theta<\infty,
$$

and

$$
B_{p, \infty}^{\Omega}=\left\{f \in L_{p}\left(\pi_{d}\right):\|f\|_{B, \infty}^{\Omega}=\sup _{s} \frac{\left\|\delta_{s}(f)\right\|_{p}}{\Omega\left(2^{-s}\right)} \leq 1\right\} .
$$

Here and below, $\Omega\left(2^{-s}\right)=\Omega\left(2^{-s_{1}}, \ldots, 2^{-s_{d}}\right), s_{j} \in \mathbb{N}, j=\overline{1, d}$.

The given definitions of the classes $B_{p, \theta}^{\Omega}$ can be extended also to the extreme values $p=1$ and $p=\infty$, by modifying the "blocks" $\delta_{s}(f)$ in (1) and (2). Let $V_{n}(t)$ stand for a Vallée-Poussin kernel of the order $2 n-1$, i.e.

$$
V_{n}(t)=1+2 \sum_{k=1}^{n} \cos k t+2 \sum_{k=n+1}^{2 n-1}\left(1-\frac{k-n}{n}\right) \cos k t .
$$

To every vector $s=\left(s_{1}, \ldots, s_{d}\right), s_{j} \in \mathbb{N}, j=\overline{1, d}$, we put in correspondence the polynomial

$$
A_{S}(x)=\prod_{j=1}^{d}\left(V_{2^{s_{j}}}\left(x_{j}\right)-V_{2^{s_{j}-1}}\left(x_{j}\right)\right) .
$$

For $f \in L_{p}\left(\pi_{d}\right), 1 \leq p \leq \infty$, by $A_{s}(f)$ we denote the convolution

$$
A_{s}(f):=A_{s}(f, x)=\left(f * A_{s}\right)(x) .
$$

Then, to within absolute constants, the classes $B_{p, \theta}^{\Omega}, 1 \leq p \leq \infty$, can be defined as follows:

$$
B_{p, \theta}^{\Omega}=\left\{f \in L_{p}\left(\pi_{d}\right):\|f\|_{B_{p, \theta}^{\Omega}}=\left(\sum_{s} \Omega^{-\theta}\left(2^{-s}\right)\left\|A_{s}(f)\right\|_{p}^{\theta}\right)^{1 / \theta} \leq 1\right\} \quad \text { for } 1 \leq \theta<\infty,
$$

and

$$
B_{p, \infty}^{\Omega}=\left\{f \in L_{p}\left(\pi_{d}\right):\|f\|_{B_{p, \infty}^{\Omega}}=\sup _{s} \frac{\left\|A_{s}(f)\right\|_{p}}{\Omega\left(2^{-s}\right)} \leq 1\right\} .
$$

We note that relations (3) and (4) were obtained in works [21] and [14], respectively. 
We note also that for $\Omega(t)=\prod_{j=1}^{d} t_{j}^{r_{j}}, 0<r_{j}<l$, the classes $B_{p, \theta}^{\Omega}$ are analogs of the wellknown Besov $B_{p, \theta}^{r}, 1 \leq \theta<\infty$, and Nikol'skii $B_{p, \infty}^{r}=H_{p}^{r}$ classes (see, e.g. [9]).

In what follows, we study the classes $B_{p, \theta}^{\Omega}$ that are defined by the function

$$
\Omega(t)=\Omega\left(t_{1}, \ldots, t_{d}\right)= \begin{cases}\prod_{j=1}^{d} \frac{t_{j}^{r}}{\left(\log \left(1 / t_{j}\right)\right)_{+}^{b_{j}}}, & \text { if } t_{j}>0, j=\overline{1, d}, \\ 0, & \text { if } \prod_{j=1}^{d} t_{j}=0 .\end{cases}
$$

Here and below, we consider the logarithms with base 2, and

$$
\left(\log \frac{1}{t_{j}}\right)_{+}=\max \left\{1, \log \frac{1}{t_{j}}\right\} .
$$

In addition, we assume that $b_{j} \in \mathbb{R}, j=\overline{1, d}$, and $0<r<l$. Hence, properties 1) -4 ) and the conditions $(S)$ and $\left(S_{l}\right)$ are satisfied for the function $\Omega(t)$ of the form (5).

In the present paper, we obtain the exact-order estimates of orthoprojective widths of the classes $B_{p, \theta}^{\Omega}$ in the space $L_{q}, 1 \leq p<q<\infty$. We recall that the notion of orthoprojective width was introduced by V.N. Temlyakov [24].

Let $\left\{u_{i}\right\}_{i=1}^{M}$ be an orthonormalized system of functions $u_{i} \in L_{\infty}\left(\pi_{d}\right), f \in L_{q}\left(\pi_{d}\right)$, $1 \leq q \leq \infty$. We set

$$
\left(f, u_{i}\right)=(2 \pi)^{-d} \int_{\pi_{d}} f(x) \bar{u}_{i}(x) d x,
$$

where $\bar{u}_{i}$ is the function complex conjugate to the function $u_{i}$.

To every function $f \in L_{q}\left(\pi_{d}\right), 1 \leq q \leq \infty$, we put in correspondence an approximation of the form

$$
\sum_{i=1}^{M}\left(f, u_{i}\right) u_{i}
$$

i.e. the orthogonal projection of the function $f$ onto the subspace generated by the system of functions $\left\{u_{i}\right\}_{i=1}^{M}$. Then for the functional class $F \subset L_{q}\left(\pi_{d}\right)$ the quantity

$$
d_{M}^{\perp}\left(F, L_{q}\right)=\inf _{\left\{u_{i}\right\}_{i=1}^{M}} \sup _{f \in F}\left\|f-\sum_{i=1}^{M}\left(f, u_{i}\right) u_{i}\right\|_{q}
$$

is called the orthoprojective width (the Fourier-width) of this class in the space $L_{q}\left(\pi_{d}\right)$.

In addition to orthoprojective widths, we study the quantities $d_{M}^{B}\left(F, L_{q}\right)$ introduced by V.N. Temlyakov (see, e.g. [23]). They are defined by

$$
d_{M}^{B}\left(F, L_{q}\right)=\inf _{G \in L_{M}(B)_{q}} \sup _{f \in F \cap D(G)}\|f-G f\|_{q} .
$$

Here, $L_{M}(B)_{q}$ stands for a set of linear operators satisfying the following conditions:

a) the domain $D(G)$ of these operators contains all trigonometric polynomials, and their range is contained in a subspace with dimension $M$ of the space $L_{q}\left(\pi_{d}\right)$;

b) there exists a number $B \geq 1$ such that for all vectors $k=\left(k_{1}, \ldots, k_{d}\right), k_{j} \in \mathbb{Z}, j=\overline{1, d}$, the inequality $\left\|G e^{i(k, \cdot)}\right\|_{2} \leq B$ holds. 
We note that $L_{M}(1)_{2}$ contains the operators of orthogonal projection onto the spaces with dimension $M$ and the operators that are set on an orthonormalized system of functions with the help of the multiplier defined by a sequence $\left\{\lambda_{m}\right\}$ such that $\left|\lambda_{m}\right| \leq 1$ for all $m$.

From (6) and (7), it is easy to see that the quantities $d_{M}^{\perp}\left(F, L_{q}\right)$ and $d_{M}^{B}\left(F, L_{q}\right)$ are connected with each other by the inequality

$$
d_{M}^{B}\left(F, L_{q}\right) \leq d_{M}^{\perp}\left(F, L_{q}\right) .
$$

At present, a lot of works are known, in which the quantities $d_{M}^{\perp}\left(F, L_{q}\right)$ and $d_{M}^{B}\left(F, L_{q}\right)$ were studied for various classes of functions. We mention works [15, 17, 18, 23, 25], where the quantities (6) and (7) were considered for the classes of functions of many variables $W_{p, \alpha}^{r}, H_{p}^{r}, B_{p, \theta}^{r}$, and $H_{p}^{\Omega}$ (see also numerous references therein). Note that the below obtained estimates complement the results established in the works $[2-4,6,8]$.

\section{Auxiliary assertions}

We now give several known assertions, which are used in the subsequent considerations.

As was noted above, $\Omega(t)$ is a function of the form (5). For natural $N$, we set

$$
\chi(N)=\left\{s=\left(s_{1}, \ldots, s_{d}\right): s_{j} \in \mathbb{N}, j=\overline{1, d}, \Omega\left(2^{-s}\right) \geq \frac{1}{N}\right\}, \quad Q(N)=\bigcup_{s \in \chi(N)} \rho(s) .
$$

We note that the approximation of certain classes of periodic functions of many variables with mixed generalized smoothness by trigonometric polynomials with "numbers" of harmonics from the sets that are analogs of $Q(N)$ was started in work [16]. Later, the approximations by trigonometric polynomials with "numbers" of harmonics from the sets $Q(N)$ were studied in works $[8,19,20]$ and other ones.

The following proposition is true.

Lemma 1 ([15]). For the number of elements of the set $Q(N)$, the following ordinal equalities hold:

$$
|Q(N)| \asymp N^{1 / r}(\log N)^{-b_{1} / r-\ldots-b_{d} / r+d-1},
$$

if $b_{1} \leq \ldots \leq b_{d}<r$

$$
|Q(N)| \asymp N^{1 / r}(\log N)^{-b_{1} / r}
$$

if $r \leq b_{1} \leq \ldots \leq b_{d}, b_{2}>r$.

Here and below, the notation $\mu_{1} \ll \mu_{2}$ for positive functions $\mu_{1}(N)$ and $\mu_{2}(N)$ means that there exists a constant $C>0$ such that for all $N \in \mathbb{N}$ the inequality $\mu_{1}(N) \leq C \mu_{2}(N)$ holds.

The relation $\mu_{1} \asymp \mu_{2}$ holds if $\mu_{1} \ll \mu_{2}$ and $\mu_{1} \gg \mu_{2}$. We note also that all constants $C_{i}$, $i=1,2, \ldots$, which are used in what follows, can depend only on parameters that are contained in the definitions of a class and a dimension $d$ of the space $\mathbb{R}^{d}$.

Let $\mathfrak{M}$ be certain finite set. By $|\mathfrak{M}|$, we denote the number of its elements.

To formulate the following assertions, we note that, according to (5), the definition of a set $\chi(N)$ takes the form

$$
\chi(N)=\left\{s=\left(s_{1}, \ldots, s_{d}\right): s_{j} \in \mathbb{N}, j=\overline{1, d}, \prod_{j=1}^{d} 2^{r s_{j}} s_{j}^{b_{j}} \leq N\right\} .
$$


Denote $\chi^{\perp}(N)=\mathbb{N}^{d} \backslash \chi(N)$.

Let

$$
\Theta(N)=\left\{s=\left(s_{1}, \ldots, s_{d}\right): s_{j} \in \mathbb{N}, j=\overline{1, d}, \frac{1}{2^{l} N} \leq \Omega\left(2^{-s}\right)<\frac{1}{N}\right\} .
$$

In work [12], it was established that the number of elements of the set $\Theta(N)$ satisfies the ordinal equality $|\Theta(N)| \asymp(\log N)^{d-1}$.

Lemma 2 (see, e.g. [23, p. 25]). Let $1 \leq p<q<\infty$ and $f \in L_{p}\left(\pi_{d}\right)$. Then

$$
\|f\|_{q}^{q} \ll \sum_{s}\left(\left\|\delta_{S}(f)\right\|_{p} 2^{\|s\|_{1}(1 / p-1 / q)}\right)^{q} .
$$

Lemma 3 ([25]). Let $\mathbb{A}$ be the linear operator given by the equality $\mathbb{A} e^{i(k, x)}=\sum_{m=1}^{\bar{M}} a_{m}^{k} \psi_{m}(x)$, where $\left\{\psi_{m}(x)\right\}_{m=1}^{\bar{M}}$ is the set of functions for which $\left\|\psi_{m}(\cdot)\right\|_{2} \leq 1, m=1, \ldots, \bar{M}$. Then for any trigonometric polynomial the following inequality holds

$$
\min _{y=x} \operatorname{Re} \mathbb{A} t(x-y) \leq \operatorname{Re} \sum_{k} \sum_{m=1}^{\bar{M}} \widehat{t}(k) a_{m}^{k} \widehat{\psi}_{m}(k) \leq\left(\bar{M} \sum_{m=1}^{\bar{M}} \sum_{k}\left|a_{m}^{k} \widehat{t}(k)\right|^{2}\right)^{1 / 2} .
$$

Theorem 1 ([11]). Let $T_{n}$ be a trigonometric polynomial of the order $n=\left(n_{1}, \ldots, n_{d}\right)$, i.e.

$$
T_{n}(x)=\sum_{\left|k_{1}\right| \leq n_{1}} \cdots \sum_{\left|k_{d}\right| \leq n_{d}} c_{k_{1}, \ldots, k_{d}} e^{i(k, x)}
$$

where $n_{j}, j=\overline{1, d}$, are natural numbers, and $c_{k_{1}, \ldots, k_{d}}$ are any coefficients.

Then for $1 \leq p<q \leq \infty$ the inequality

$$
\left\|T_{n}\right\|_{q} \leq 2^{d}\left(\prod_{j=1}^{d} n_{j}\right)^{1 / p-1 / q}\left\|T_{n}\right\|_{p}
$$

holds.

Inequality (9) was established by S.M. Nikol'skii and is called the "inequality of different metrics". In the one-dimensional case for $p=\infty$, the corresponding inequality was proved by D. Jackson [7].

Theorem 2 (Littlewood-Paley theorem; see, e.g. [10, p. 65]). Let $p \in(1, \infty)$. Then there exist positive numbers $C_{3}(p)$ and $C_{4}(p)$ such that for every function $f \in L_{p}\left(\pi_{d}\right)$ the following relations are true

$$
C_{3}(p)\|f\|_{p} \leq\left\|\left(\sum_{S}\left|\delta_{S}(f)\right|^{2}\right)^{1 / 2}\right\|_{p} \leq C_{4}(p)\|f\|_{p}
$$

\section{Main results}

Passing to the statement of the propositions and their proofs, we assume that $M=|Q(N)|$. First, we consider case $b_{1} \leq \ldots \leq b_{d}<r$. Then, according to Lemma 1 , we have

$$
M \asymp N^{1 / r}(\log N)^{-b_{1} / r-\ldots-b_{d} / r+d-1}, \quad \log M \asymp \log N, \quad N \asymp M^{r}(\log M)^{b_{1}+\ldots+b_{d}-(d-1) r} .
$$

The following theorem is true. 
Theorem 3. Let $1 \leq p<q<\infty, 1 \leq \theta \leq q$, and let $\Omega(t)$ be a function of the form (5). Then for $1 / p-1 / q<r<l, b_{1} \leq \ldots \leq b_{d}<r /(q / p-1)$ the relations

$$
d_{M}^{\perp}\left(B_{p, \theta}^{\Omega}, L_{q}\right) \asymp d_{M}^{B}\left(B_{p, \theta}^{\Omega}, L_{q}\right) \asymp M^{-r+1 / p-1 / q}(\log M)^{-b_{1}-\ldots-b_{d}+(d-1)(r-1 / p+1 / q)}
$$

hold.

Proof. First, we establish the upper bounds in (10). According to (8), it is sufficient to obtain the upper bound for the orthoprojective width $d_{M}^{\perp}\left(B_{p, \theta^{\prime}}^{\Omega} L_{q}\right)$.

For this purpose, we consider an approximation of the functions $f \in B_{p, \theta}^{\Omega}$ by trigonometric polynomials $t_{Q(N)}$ of the form $t_{Q(N)}(x)=\sum_{s \in \chi(N)} \delta_{s}(f, x)$.

Let $q_{0}$ be any number that satisfies the condition $p<q_{0}<q$. Then, using Lemma 2 , and the relation $\left\|\delta_{s}(f)\right\|_{q_{0}} \asymp\left\|A_{s}(f)\right\|_{q_{0}}, 1<q_{0}<\infty$, for $f \in B_{p, \theta}^{\Omega}$ we have

$$
\begin{aligned}
\left\|f-t_{Q(N)}\right\|_{q} & =\left\|f-\sum_{s \in \chi(N)} \delta_{s}(f)\right\|_{q}=\left\|\sum_{s \in \chi^{\perp}(N)} \delta_{s}(f)\right\|_{q} \\
& \ll\left(\sum_{s \in \chi^{\perp}(N)}\left\|\delta_{s}(f)\right\|_{q_{0}}^{q} 2^{\|s\|_{1}\left(1 / q_{0}-1 / q\right) q}\right)^{1 / q} \\
& \asymp\left(\sum_{s \in \chi^{\perp}(N)}\left\|A_{s}(f)\right\|_{q_{0}}^{q} 2^{\|s\|_{1}\left(1 / q_{0}-1 / q\right) q}\right)^{1 / q}=I_{1} .
\end{aligned}
$$

Then, applying to $A_{s}(f)$ the Nikol'skii inequality of different metrics (9), we continue the estimate as follows

$$
\begin{aligned}
I_{1} & \ll\left(\sum_{s \in \chi^{\perp}(N)}\left\|A_{s}(f)\right\|_{p}^{q} 2^{\|s\|_{1}\left(1 / p-1 / q_{0}\right) q} 2^{\|s\|_{1}\left(1 / q_{0}-1 / q\right) q}\right)^{1 / q} \\
& =\left(\sum_{s \in \chi^{\perp}(N)}\left\|A_{s}(f)\right\|_{p}^{q} 2^{\|s\|_{1}(1 / p-1 / q) q}\right)^{1 / q} \\
& =\left(\sum_{s \in \chi^{\perp}(N)} \Omega^{-q}\left(2^{-s}\right)\left\|A_{s}(f)\right\|_{p}^{q} \Omega^{q}\left(2^{-s}\right) 2^{\|s\|_{1}(1 / p-1 / q) q}\right)^{1 / q} \\
& \left.=\left(\sum_{s \in \chi^{\perp}(N)} \Omega^{-q}\left(2^{-s}\right) \| A_{s}(f)\right) \|_{p}^{q} \frac{2^{-q\|s\|_{1}(r-1 / p+1 / q)}}{\prod_{j=1}^{d} s_{j}^{q b_{j}}}\right)^{1 / q}=I_{2} .
\end{aligned}
$$

If $s \in \chi^{\perp}(N)$, then $2^{-\|s\|_{1}}<N^{-1 / r} \prod_{j=1}^{d} s_{j}^{b_{j} / r}$.

We get

$$
\begin{aligned}
I_{2} & <\left(\sum_{s \in \chi^{\perp}(N)} \Omega^{-q}\left(2^{-s}\right)\left\|A_{s}(f)\right\|_{p}^{q} N^{-q / r(r-1 / p+1 / q)} \prod_{j=1}^{d} \frac{s_{j}^{q b_{j} / r(r-1 / p+1 / q)}}{s_{j}^{q b_{j}}}\right)^{1 / q} \\
& =N^{-1+1 / r(1 / p-1 / q)}\left(\sum_{s \in \chi^{\perp}(N)} \Omega^{-q}\left(2^{-s}\right)\left\|A_{s}(f)\right\|_{p}^{q} \prod_{j=1}^{d} s_{j}^{-q b_{j} / r(1 / p-1 / q)}\right)^{1 / q} \\
& \ll N^{-1+1 / r(1 / p-1 / q)}(\log N)^{\left(-b_{1} / r-\ldots-b_{d} / r\right)(1 / p-1 / q)}\left(\sum_{s \in \chi^{\perp}(N)} \Omega^{-q}\left(2^{-s}\right)\left\|A_{s}(f)\right\|_{p}^{q}\right)^{1 / q}=I_{3} .
\end{aligned}
$$


Using the inequality (see, e.g. [5, p. 43])

$$
\left(\sum_{k}\left|a_{k}\right|^{v_{2}}\right)^{1 / v_{2}} \leq\left(\sum_{k}\left|a_{k}\right|^{v_{1}}\right)^{1 / v_{1}}, \quad 1 \leq v_{1} \leq v_{2}<\infty,
$$

we have

$$
\begin{aligned}
I_{3} & \leq N^{-1+1 / r(1 / p-1 / q)}(\log N)^{\left(-b_{1} / r-\ldots-b_{d} / r\right)(1 / p-1 / q)}\left(\sum_{s \in \chi^{\perp}(N)} \Omega^{-\theta}\left(2^{-s}\right)\left\|A_{s}(f)\right\|_{p}^{\theta}\right)^{1 / \theta} \\
& \ll N^{-1+1 / r(1 / p-1 / q)}(\log N)^{\left(-b_{1} / r-\ldots-b_{d} / r\right)(1 / p-1 / q)}\|f\|_{B_{p, \theta}^{\Omega}} \\
& \ll\left(M^{r}(\log M)^{b_{1}+\ldots+b_{d}-(d-1) r}\right)^{-1+1 / r(1 / p-1 / q)}(\log M)^{\left(-b_{1} / r-\ldots-b_{d} / r\right)(1 / p-1 / q)} \\
& =M^{-r+1 / p-1 / q}(\log M)^{-b_{1}-\ldots-b_{d}+(d-1)(r-1 / p+1 / q)} .
\end{aligned}
$$

Thus, in view of the definition of orthoprojective width, the above reasoning gives the upper bound for $d_{M}^{\perp}\left(B_{p, \theta}^{\Omega}, L_{q}\right)$, and, respectively, for the quantity $d_{M}^{B}\left(B_{p, \theta}^{\Omega}, L_{q}\right)$.

Let us find the lower bounds in (10). Since inequality (8) holds, it is sufficient to obtain the lower bound for the quantity $d_{M}^{B}\left(B_{p, \theta}^{\Omega}, L_{q}\right)$.

With the help of the reasoning analogous to that in [13], we can prove the existence of a set $\Theta_{1}(N) \subset \Theta(N)$ such that for $s=\left(s_{1}, \ldots, s_{d}\right) \in \Theta_{1}(N)$ the following relations are satisfied

$$
s_{j} \asymp \log N, \quad j=\overline{1, d}, \quad \text { and } \quad\left|\Theta_{1}(N)\right| \asymp(\log N)^{d-1} .
$$

Consider the set $\widetilde{Q}(N)=\bigcup_{s \in \Theta_{1}(N)} \rho(s)$. By $T(\widetilde{Q}(N))$ we denote the set of trigonometric polynomials with the "numbers" of harmonics from $\widetilde{Q}(N)$.

Let $K_{n}$ be the Fejér kernel of the order $n$, i.e.

$$
K_{n}(t)=\sum_{|k| \leq n}\left(1-\frac{|k|}{n+1}\right) e^{i k x} .
$$

We denote by $k^{s}$ the vector $k^{s}=\left(k_{1}^{s_{1}}, \ldots, k_{d}^{s_{d}}\right)$, where

$$
k_{j}^{s_{j}}= \begin{cases}2^{s_{j}-1}+2^{s_{j}-2}, & s_{j} \geq 2, \\ 1, & s_{j}=1, j=\overline{1, d} .\end{cases}
$$

Consider the function $g(x)=\sum_{s \in \Theta_{1}(N)} \mathcal{K}^{s}(x)$, where $\mathcal{K}^{s}(x)=e^{i\left(k^{s}, x\right)} \prod_{j=1}^{d} K_{2^{s} j^{-2}}\left(x_{j}\right)$.

Suppose that the operator $G$ belongs to $L_{M}(B)_{q}, 1<q<\infty$. Consider the operator $\mathbb{A}=$ $S_{\widetilde{Q}(N)} G$, where $S_{\widetilde{Q}(N)}$ is the operator of taking partial Fourier sum corresponding to the set $\widetilde{Q}(N)$. Then $\mathbb{A} \in L_{M}(B)_{q}$ and the range of the operator $\mathbb{A}$ is a subspace $\mathcal{L}_{M}$ of the space $T(\widetilde{Q}(N))$, whose dimension $\operatorname{dim} \mathcal{L}_{M}$ satisfies $\operatorname{dim} \mathcal{L}_{M}=\bar{M} \leq M$. It follows from Theorem 2 that for $f \in T(\widetilde{Q}(N))$, the relation $\|f-\mathbb{A} f\|_{q} \ll\|f-G f\|_{q}$ is satisfied.

Let $\left\{\psi_{m}(x)\right\}_{m=1}^{\bar{M}}$ be an orthonormal basis in $\mathcal{L}_{M}$ and $\mathbb{A} e^{i(k, x)}=\sum_{m=1}^{\bar{M}} a_{m}^{k} \psi_{m}(x)$. Consider for $s \in \Theta_{1}(N)$ the operators

$$
\mathbb{A}_{s} e^{i(k, x)}=\sum_{m=1}^{\bar{M}} a_{m}^{k} \delta_{s}\left(\psi_{m}, x\right), \quad k \in \widetilde{Q}(N) .
$$


We put $g_{s}(x)=\mathcal{K}^{s}(x)$ and consider the quantities

$$
I_{s}=\sup _{y}\left\|g_{s}(x-y)-\mathbb{A}_{s}\left(g_{s}(x-y)\right)\right\|_{\infty}, \quad s \in \Theta_{1}(N) .
$$

We denote $\beta_{s}=\min _{y=x} \operatorname{Re} \mathbb{A}_{s}\left(g_{s}(x-y)\right)$. Then

$$
I_{s} \geq g_{s}(0)-\beta_{s}
$$

Next, using Lemma 3 we have

$$
\beta_{s} \leq \operatorname{Re} \sum_{|k| \in \rho(s)} \sum_{m=1}^{\bar{M}} \widehat{g}_{s}(k) a_{m}^{k} \widehat{\psi}_{m}(k), \quad s \in \Theta_{1}(N),
$$

where we get that

$$
\begin{aligned}
\sum_{s \in \Theta_{1}(N)} \beta_{s} & \leq \operatorname{Re} \sum_{m=1}^{\bar{M}} \sum_{k \in \widetilde{Q}(N)} \widehat{g}(k) a_{m}^{k} \widehat{\psi}_{m}(k) \leq\left(\bar{M} \sum_{m=1}^{\bar{M}} \sum_{k \in \widetilde{Q}(N)}\left|a_{m}^{k} \widehat{g}(k)\right|^{2}\right)^{1 / 2} \\
& \leq M^{1 / 2} B\left(\sum_{k \in \widetilde{Q}(N)}|\widehat{g}(k)|^{2}\right)^{1 / 2} \ll M^{1 / 2} B|\widetilde{Q}(N)|^{1 / 2}
\end{aligned}
$$

Further, taking into account the relation $\left|\Theta_{1}(N)\right| \asymp(\log N)^{d-1}$ as well as

$$
|\rho(s)| \asymp 2^{\|s\|_{1}} \asymp N^{1 / r}(\log N)^{-b_{1} / r-\ldots-b_{d} / r},
$$

we can write

$$
|\widetilde{Q}(N)| \asymp N^{1 / r}(\log N)^{-b_{1} / r-\ldots-b_{d} / r+d-1} .
$$

On the other hand,

$$
g(0) \asymp N^{1 / r}(\log N)^{-b_{1} / r-\ldots-b_{d} / r+d-1} \asymp|\widetilde{Q}(N)| .
$$

Using (12) and (13), we can chose a number $N$ such that $|\widetilde{Q}(N)| \asymp M$ and the right-hand side of (14) will be at least twice as large as the right-hand side of (12).

Since $g(0)=\sum_{s \in \Theta_{1}(N)} g_{s}(0)$, from (12) and (14) we obtain that there exists an $s^{*} \in \Theta_{1}(N)$ for which

$$
g_{s^{*}}(0)-\beta_{s^{*}} \geq \frac{B(M|\widetilde{Q}(N)|)^{1 / 2}}{\left|\Theta_{1}(N)\right|} \asymp M(\log N)^{-(d-1)} .
$$

Then from (11), using the Nikolskii inequality of different metrics, we have that for some $y^{*}$ the following relations hold

$$
\begin{aligned}
\left\|\mathcal{K}^{s^{*}}\left(x-y^{*}\right)-\mathbb{A}_{s^{*}}\left(\mathcal{K}^{s^{*}}\left(x-y^{*}\right)\right)\right\|_{q} & =\left\|g_{s^{*}}\left(x-y^{*}\right)-\mathbb{A}_{s^{*}}\left(g_{s^{*}}\left(x-y^{*}\right)\right)\right\|_{q} \\
& \gg 2^{-\left\|s^{*}\right\|_{1} / q}\left\|g_{s^{*}}\left(x-y^{*}\right)-\mathbb{A}_{s^{*}}\left(g_{s^{*}}\left(x-y^{*}\right)\right)\right\|_{\infty} \\
& \asymp 2^{-\left\|s^{*}\right\|_{1} / q} M(\log N)^{-(d-1)} \asymp M^{1-1 / q}(\log M)^{-(d-1)(1-1 / q) .}
\end{aligned}
$$

Consider the function $g_{1}(x)=C_{5} N^{-1}\left(N^{1 / r}(\log N)^{-b_{1} / r-\ldots-b_{d} / r}\right)^{1 / p-1} \mathcal{K}^{s^{*}}(x), C_{5}>0$.

We can easily verify that the function $g_{1}(x)$ belongs to the class $B_{p, \theta^{\prime}}^{\Omega}$, under the corresponding choice of the constant $C_{5}$. 
Using the Fejér kernel property (see, e.g. $\left[23\right.$, p. 166]) $\left\|\mathcal{K}^{s^{*}}\right\|_{p} \asymp 2^{\left\|s^{*}\right\|_{1}(1-1 / p)}, 1 \leq p \leq \infty$, we have

$$
\begin{aligned}
\left\|A_{S^{*}}\left(g_{1}\right)\right\|_{p} & \ll N^{-1}\left(N^{1 / r}(\log N)^{-b_{1} / r-\ldots-b_{d} / r}\right)^{1 / p-1}\left\|\mathcal{K}^{s^{*}}\right\|_{p} \\
& \asymp N^{-1}\left(N^{1 / r}(\log N)^{-b_{1} / r-\ldots-b_{d} / r}\right)^{1 / p-1} 2^{\left\|s^{*}\right\|_{1}(1-1 / p)} \\
& \asymp N^{-1}\left(N^{1 / r}(\log N)^{-b_{1} / r-\ldots-b_{d} / r}\right)^{1 / p-1}\left(N^{1 / r}(\log N)^{-b_{1} / r-\ldots-b_{d} / r}\right)^{1-1 / p}=N^{-1} .
\end{aligned}
$$

Thus, we can write

$$
\left\|g_{1}\right\|_{B_{p, \theta}^{\Omega}}=\left(\sum_{s} \Omega^{-\theta}\left(2^{-s}\right)\left\|A_{s}\left(g_{1}\right)\right\|_{p}^{\theta}\right)^{1 / \theta}=\Omega^{-1}\left(2^{-s^{*}}\right)\left\|A_{s^{*}}\left(g_{1}\right)\right\|_{p} \ll \Omega^{-1}\left(2^{-s^{*}}\right) N^{-1} \asymp 1 .
$$

So, we conclude that $g_{1} \in B_{p, \theta}^{\Omega}$, with the corresponding constant $C_{5}>0$.

Since $\left\|g_{1}-G g_{1}\right\|_{q} \gg\left\|g_{1}-\mathbb{A}_{s^{*}} g_{1}\right\|_{q}$, we get

$$
\begin{aligned}
\| g_{1}\left(x-y^{*}\right) & -G g_{1}\left(x-y^{*}\right) \|_{q} \\
& \gg N^{-1}\left(N^{1 / r}(\log N)^{-b_{1} / r-\ldots-b_{d} / r}\right)^{1 / p-1}\left\|\mathcal{K}^{s^{*}}\left(x-y^{*}\right)-\mathbb{A}_{s^{*}}\left(\mathcal{K}^{s^{*}}\left(x-y^{*}\right)\right)\right\|_{q} \\
& \gg M^{-r}(\log M)^{-b_{1}-\ldots-b_{d}+(d-1) r} M^{1 / p-1} M^{1-1 / q}(\log M)^{(d-1)(1-1 / p)-(d-1)(1-1 / q)} \\
& =M^{-r+1 / p-1 / q}(\log M)^{-b_{1}-\ldots-b_{d}+(d-1)(r-1 / p+1 / q)} .
\end{aligned}
$$

The lower bounds in (10) are established.

In the following proposition, we consider other relations for the numbers $r, b_{1}, \ldots, b_{d}$. Let $r \leq b_{1} \leq \ldots \leq b_{d}, b_{2}>r$. In this case, by Lemma 1 , we obtain

$$
M \asymp N^{1 / r}(\log N)^{-b_{1} / r}, \quad \log M \asymp \log N, \quad N \asymp M^{r}(\log M)^{b_{1}} .
$$

Assume that $b_{1}=\ldots=b_{v}<b_{v+1} \leq \ldots \leq b_{d}$. Then for $v=1$ the inequality $r \leq b_{1}<b_{2}$ holds. But $v \geq 2$, then $b_{1}>r$.

Theorem 4. Let $1 \leq p<q<\infty, 1 \leq \theta \leq q$, and let $\Omega(t)$ be a function of the form (5). Then for $1 / p-1 / q<r<l, b_{2}>r /(q / p-1)$ the order estimates

$$
d_{M}^{\perp}\left(B_{p, \theta}^{\Omega}, L_{q}\right) \asymp d_{M}^{B}\left(B_{p, \theta}^{\Omega}, L_{q}\right) \asymp M^{-r+1 / p-1 / q}(\log M)^{-b_{1}}
$$

hold.

Proof. For $1 \leq \theta \leq q$, the embedding $B_{p, \theta}^{\Omega} \subset H_{p}^{\Omega}$ is valid. Therefore, the upper bounds in (15) follow from the corresponding estimate $d_{M}^{\perp}\left(H_{p}^{\Omega}, L_{q}\right)$, proved in [15].

To get the lower bounds in (15), it is sufficient to get the corresponding lower bound for the quantity $d_{M}^{B}\left(B_{p, \theta}^{\Omega}, L_{q}\right)$.

We choose a vector $\tilde{s}=\left(\tilde{s}_{1}, \ldots, \tilde{s}_{d}\right) \in \Theta(N)$, so, that $\tilde{s}_{1} \asymp \log N, \tilde{s}_{2}=\ldots=\tilde{s}_{d}=1$, and set $g_{2}(x)=\mathcal{K}_{\tilde{s}}(x)=e^{i\left(k^{\tilde{s}}, x\right)} K_{2^{\tilde{s}_{1}-2}}\left(x_{1}\right)$, where $k^{\tilde{s}}=\left(2^{\tilde{s}_{1}-1}+2^{\tilde{s}_{1}-2}, 1, \ldots, 1\right)$.

Suppose that the operator $G$ belongs to $L_{M}(B)_{q}, 1<q<\infty$. Consider the operator $\mathbb{A}=S_{\rho(\tilde{s})} G$, where $S_{\rho(\tilde{s})}$ is the operator of taking partial Fourier sum corresponding to the $\operatorname{set} \rho(\tilde{s})$.

Taking into account that $2^{\|\tilde{s}\|_{1}} \asymp N^{1 / r}(\log N)^{-b_{1} / r}$, and using lemma 3 , we get

$$
\begin{aligned}
\min _{y=x} \operatorname{Re} \mathbb{A} g_{2}(x-y) & \leq M^{1 / 2} B\left(\sum_{k}\left|\widehat{g}_{2}(k)\right|^{2}\right)^{1 / 2} \\
& \ll M^{1 / 2}\left(2^{\|\tilde{s}\|_{1}}\right)^{1 / 2} \asymp M^{1 / 2}\left(N^{1 / r}(\log N)^{-b_{1} / r}\right)^{1 / 2} .
\end{aligned}
$$


On the other hand,

$$
g_{2}(0) \asymp 2^{\|\tilde{s}\|_{1}} \asymp N^{1 / r}(\log N)^{-b_{1} / r} .
$$

Therefore, we can chose a number $N$ so that $|Q(N)| \asymp M$ and the right-hand side of (17) will be at least twice as large as the right-hand side of (16). For some $y^{*}=\left(y_{1}^{*}, \ldots, y_{d}^{*}\right)$, for this $N$ we have

$$
\left\|g_{2}\left(x-y^{*}\right)-\mathbb{A} g_{2}\left(x-y^{*}\right)\right\|_{\infty} \gg M .
$$

Consider the function $g_{3}(x)=C_{6} N^{-1} 2^{\|\tilde{s}\|_{1}(1 / p-1)} g_{2}(x), C_{6}>0$. We now show that, at the corresponding choice of the constant $C_{6}$, the function $g_{3}$ belongs to the class $B_{p, \theta}^{\Omega}$.

Indeed, in view of the properties of the Fejér kernel, we have

$$
\begin{aligned}
\left\|g_{3}\right\|_{B_{p, \theta}^{\Omega}}=\left(\sum_{s} \Omega^{-\theta}\left(2^{-s}\right)\left\|A_{s}\left(g_{3}\right)\right\|_{p}^{\theta}\right)^{1 / \theta} & \ll N^{-1} 2^{\|\tilde{s}\|_{1}(1 / p-1)}\left(\Omega^{-\theta}\left(2^{-\tilde{s}}\right)\left\|A_{\tilde{s}}\left(g_{2}\right)\right\|_{p}^{\theta}\right)^{1 / \theta} \\
& \ll 2^{\|\tilde{s}\|_{1}(1 / p-1)}\left\|A_{\tilde{s}}\left(g_{2}\right)\right\|_{p} \asymp 2^{\|\tilde{s}\|_{1}(1 / p-1)} 2^{\|\tilde{s}\|_{1}(1-1 / p)}=1 .
\end{aligned}
$$

Hence, $g_{3} \in B_{p, \theta}^{\Omega}$ with the corresponding constant $C_{6}>0$.

According to Theorem 1, for a trigonometric polynomial $t$ with "numbers" of harmonics from the set $\rho(\tilde{s})$, the relation $\|t\|_{\infty} \ll\|t\|_{q} 2^{\|\tilde{s}\|_{1} / q}$ is satisfied.

Taking into account the last relation and using estimate (18), we get

$$
\begin{aligned}
\left\|g_{3}\left(x-y^{*}\right)-G g_{3}\left(x-y^{*}\right)\right\|_{q} & \gg N^{-1} 2^{\|\tilde{s}\|_{1}(1 / p-1)}\left\|g_{2}\left(x-y^{*}\right)-G g_{2}\left(x-y^{*}\right)\right\|_{q} \\
& \gg N^{-1} 2^{\|\tilde{s}\|_{1}(1 / p-1)}\left\|g_{2}\left(x-y^{*}\right)-\mathbb{A} g_{2}\left(x-y^{*}\right)\right\|_{q} \\
& \gg N^{-1} 2^{\|\tilde{s}\|_{1}(1 / p-1)} 2^{-\|\tilde{s}\|_{1} / q}\left\|g_{2}\left(x-y^{*}\right)-\mathbb{A} g_{2}\left(x-y^{*}\right)\right\|_{\infty} \\
& \gg M^{-r}(\log M)^{-b_{1}} M^{1 / p-1 / q-1} M=M^{-r+1 / p-1 / q}(\log M)^{-b_{1}}
\end{aligned}
$$

The lower bounds in (15) are established.

At the end of the work we make some comments, paying attention to two important issues.

The first of them is that under the conditions considered in Theorems 3 and 4 for the parameter $\theta$, the obtained estimates of the approximation characteristics are independent of the parameter $\theta$. In addition, the estimates obtained in Theorem 4 coincide in order with the estimates of the corresponding characteristics for the classes $H_{p}^{\Omega}$, i.e.

$$
d_{M}^{\perp}\left(B_{p, \theta}^{\Omega}, L_{q}\right) \asymp d_{M}^{B}\left(B_{p, \theta}^{\Omega}, L_{q}\right) \asymp d_{M}^{\perp}\left(H_{p}^{\Omega}, L_{q}\right) \asymp d_{M}^{B}\left(H_{p}^{\Omega}, L_{q}\right) .
$$

Another, but not less important issue, concerns Theorem 4, in which it is found that the established estimates of the corresponding characteristics do not depend on the dimension $d$ of the space $\mathbb{R}^{d}$.

\section{References}

[1] Bari N.K., Stechkin S.B. The best approximations and differential properties of two conjugate functions. Trans. Moscow Math. Soc. 1956, 5, 483-522. (in Russian)

[2] Fedunyk-Yaremchuk O.V., Hembars'ka S.B. Estimates of approximative characteristics of the classes $B_{p, \theta}^{\Omega}$ of periodic functions of several variables with given majorant of mixed moduli of continuity in the space $L_{q}$. Carpathian Math. Publ. 2019, 11 (2), 281-295. doi:10.15330/cmp.11.2.281-295 
[3] Fedunyk-Yaremchuk O.V., Hembars'kyi M.V., Hembars'ka S.B. Approximative characteristics of the Nikol'skiiBesov-type classes of periodic functions in the space $B_{\infty, 1}$. Carpathian Math. Publ. 2020, 12 (2), $376-391$. doi:10.15330/cmp.12.2.376-391

[4] Fedunyk-Yaremchuk O.V., Solich K.V. Estimates of approximative characteristics of the classes $B_{p, \theta}^{\Omega}$ of periodic functions of many variables with given majorant of mixed continuity moduli in the space $L_{\infty}$. J. Math. Sci. (N.Y.) 2018, 231 (1), 28-40. doi:10.1007/s10958-018-3803-3 (translation of Ukr. Mat. Visn. 2017, 14 (3), 345-360. (in Ukrainian))

[5] Hardy G.H., Littlewood J.E., Polya G. Inequalities. Cambridge Univ. Press, Cambridge, 1934.

[6] Hembars'ka S.B., Fedunyk-Yaremchuk O.V. Approximation characteristics of the Nikol'sky-Besov-type classes of periodic single- and multivariable functions in the $B_{1,1}$ space. J. Math. Sci. (N.Y.) 2021, 259 (1), $75-87$. doi:10.1007/s10958-021-05600-2 (translation of Ukr. Mat. Visn. 2021, 18 (3), 289-405. (in Ukrainian))

[7] Jackson D. Certain problem of closest approximation. Bull. Amer. Math. Soc. 1933, 39, 889-906.

[8] Konograi A.F. Estimates of the approximation characteristics of the classes $B_{p, \theta}^{\Omega}$ of periodic functions of several variables with given majorant of mixed moduli of continuity. Math. Notes 2014, 95 (5), 656-189. doi: 10.1134/S0001434614050095 (translation of Mat. Zametki 2014, 95 (5), 734-749. doi:10.4213/mzm10118 (in Russian))

[9] Lizorkin P.I., Nikol'skii S.M. Spaces of functions with mixed smoothness from the decomposition point of view. Proc. Steklov Inst. Math. 1990, 187, 163-184. (translation of Tr. Mat. Inst. Steklova 1989, 187, 143-161. (in Russian))

[10] Nikol'skii S.M. Approximation of Functions of Several Variables and Embedding Theorems. Nauka, Moscow, 1969. (in Russian)

[11] Nikol'skii S.M. Inequalities for entire functions of finite degree and their application in the theory of differentiable functions of severaly variables. Tr. Mat. Inst. Steklova 1951, 38, 244-278. (in Russian)

[12] Pustovoitov N.N. Approximation of multidimensional functions with a given majorant of mixed moduli of continuity. Math. Notes 1999, 65 (1), 89-98. doi:10.1007/BF02675013 (translation of Mat. Zametki 1999, 65 (1), $107-117$. doi:10.4213/mzm1032 (in Russian))

[13] Pustovoitov N.N. On the approximation and characterization of periodic functions of many variables, whose majorant of mixed continuity moduli has a special form. Anal. Math. 2003, 29, 201-218. doi:10.1023/ A:1025415204826 (in Russian)

[14] Pustovoitov N.N. Representation and approximation of periodic functions of several variables with given mixed modulus of continuity. Anal. Math. 1994, 20, 35-48. doi:10.1007/BF01908917 (in Russian)

[15] Pustovoitov N.N. The orthowidths of classes of multidimensional periodic functions, for which the majorant of mixed continuity moduli contains power and logarithmic multipliers. Anal. Math. 2008, 34, 187-224. doi:10.1007/s10476008-0303-6 (in Russian)

[16] Romanyuk A.S. Approximation of classes of periodic functions of several variables. Ukrainian Math. J. 1992, 44 (5), 596-606. doi:10.1007/BF01056698 (translation of Ukrain. Mat. Zh. 1992, 44 (5), 662-672. (in Russian))

[17] Romanyuk A.S. Best approximations and widths of classes of periodic functions of several variables. Math. Sb. 2008, 199 (2), 253-275. doi:10.1070/SM2008v199n02ABEH003918 (translation of Math. Sb. 2008, 199 (2), 93-114. doi:10.4213/sm3685 (in Russian))

[18] Romanyuk A.S. Diameters and best approximation of the classes $B_{p, \theta}^{r}$ of periodic functions of several variables. Anal. Math. 2011, 37, 181-213. doi:10.1007/s10476-011-0303-9 (in Russian)

[19] Stasyuk S.A. Approximation by Fourier sums and the Kolmogorov widths for the classes $M B_{p, \theta}^{\Omega}$ of periodic functions of several variables. Tr. Inst. Mat. Mekh. 2014, 20 (1), 247-257. (in Russian) 
[20] Stasyuk S.A. Best approximations of periodic functions of several variables from the classes $B_{p, \theta}^{\Omega}$. Math. Notes 2010, 87 (1-2), 102-114. doi:10.1134/S000143461001013X (translation of Mat. Zametki 2010, 87 (1), $108-121$. doi:10.4213/mzm4053 (in Russian))

[21] Stasyuk S.A., Fedunyk O.V. Approximation characteristics of the classes $B_{p, \theta}^{\Omega}$ of periodic functions of many variables. Ukrainian Math. J. 2006, 58 (5), 779-793. doi:10.1007/s11253-006-0101-x (translation of Ukrain. Mat. Zh. 2006, 58 (5), 692-704. (in Ukrainian))

[22] Yongsheng S., Heping W. Representation and approximation of multivariate periodic functions with bounded mixed moduli of smoothness. Tr. Mat. Inst. Steklova 1997, 219, 356-377.

[23] Temlyakov V.N. Approximation of functions with bounded mixed derivative. Proc. Steklov Inst. Math. 1989, 178, 1-121. (translation of Tr. Mat. Inst. Steklova 1986, 178, 3-113. (in Russian))

[24] Temlyakov V.N. Diameters of some classes of functions of several variables. Dokl. Akad. Nauk SSSR 1982,267 (2), 314-317. (in Russian)

[25] Temlyakov V.N. Estimates of the asymptotic characteristics of classes of functions with bounded mixed derivative or difference. Proc. Steklov Inst. Math. 1990, 189, 161-197. (translation of Tr. Mat. Inst. Steklova 1989, 189, $138-168$. (in Russian))

Received 02.04.2021

Revised 03.12.2021

Федуник-Яремчук О.В., Гембарська С.Б. Наближення класів періодииних функиій багатьох змінних із заданою мажсорантою мішаних модулів неперервності // Карпатські матем. публ. — 2021. — T.13, №3. - C. 838-850.

У роботі продовжується вивчення апроксимативних характеристик класів $B_{p, \theta}^{\Omega}$ періодичних функцій багатьох змінних, мажоранта мішаних модулів неперервності яких містить як степеневі, так і логарифмічні множники. Одержано точні за порядком оцінки ортопроекційних поперечників класів $B_{p, \theta}^{\Omega}$ у просторі $L_{q}, 1 \leq p<q<\infty$, а також встановлено точні за порялком оцінки наближення цих класів функцій у просторі $L_{q}$ за допомогою лінійних операторів, які підпорядковані певним умовам.

Ключові слова і фрази: ортопроекційний поперечник, мішаний модуль неперервності, лінійний оператор, ялро Валле-Пуссена, ялро Фейєра. 